\title{
Numerical Study of High-Temperature Jet Flow Using RANS/LES and PANS Formulations
}

\author{
Khaled S. Abdol-Hamid* \\ NASA Langley Research Center, Hampton, VA 23681 \\ and \\ Alaa Elmiligui ${ }^{\dagger}$ \\ Analytical Services \& Materials, Inc., Hampton, VA 23666
}

\begin{abstract}
Two multi-scale-type turbulence models are implemented in the PAB3D solver. The models are based on modifying the Reynolds Averaged Navier-Stokes (RANS) equations. The first scheme is a hybrid RANS/LES model utilizing the two-equation $(\mathrm{k} \varepsilon)$ model with a RANS/LES transition function dependent on grid spacing and the computed turbulence length scale. The second scheme is a modified version of the Partially Averaged Navier-Stokes (PANS) model, where the unresolved kinetic energy parameter $\left(f_{k}\right)$ is allowed to vary as a function of grid spacing and the turbulence length scale. This parameter is estimated based on a novel two-stage procedure to efficiently estimate the level of scale resolution possible for a given flow on a given grid for Partial Averaged Navier-Stokes (PANS). It has been found that the prescribed scale resolution can play a major role in obtaining accurate flow solutions. The parameter $f_{k}$ varies between zero and one and equal to one in the viscous sub layer, and when the RANS turbulent viscosity becomes smaller than the LES viscosity. The formulation, usage methodology, and validation examples are presented to demonstrate the enhancement of PAB3D's time-accurate and turbulence modeling capabilities. The accurate simulations of flow and turbulent quantities will provide valuable tool for accurate jet noise predictions. Solutions from these models are compared to RANS results and experimental data for high-temperature jet flows. The current results show promise for the capability of hybrid RANS/LES and PANS in simulating such flow phenomena.
\end{abstract}

\section{Introduction}

$\mathrm{T}$ he limited capability of the Reynolds Averaged Navier-Stokes (RANS) approach, combined with eddy-viscosity turbulence models to simulate unsteady and complex flows, has been known for some time. The RANS assumption is that most of the energy is modeled through the turbulence transport equations and is resolved in the grid. RANS overpredicts the eddy viscosity, which results in excessive damping of unsteady motion. Consequently, the eddy viscosity attains un-physically large values due to unresolved scales and suppresses most temporal and spatial fluctuations in the resolved flow field. One of the approaches used to overcome this problem is to provide a mechanism for the RANS equations to resolve the largest scales of motion. Among several methods the Detached Eddy Simulations (DES) [1], the hybrid Large Eddy Simulation (LES) [2-3], the Limited Numerical Scheme (LNS) [4] and the Partial Averaged Navier-Stokes (PANS) [5] provide the mechanisms needed to satisfy this requirement.

In an attempt to increase the fidelity and accuracy of the PAB3D code [6-8], a hybrid turbulence model RANS/LES [2-3] and PANS [9] have been added Abdol-Hamid and Girimaji [9] explored a new approach to improve the accuracy and robustness of PANS in creating a simulation of an unsteady flow field. They accomplished this through the development and implementation of a two-stage procedure to efficiently estimate the level of scale resolution possible for a given flow on a given grid for PANS and other hybrid models. This capability was implemented in a general applied aerodynamics Computational Fluid Dynamics (CFD) research code, PAB3D, for the simulation of unsteady flows.

Both Large temperature and pressure fluctuations have a profound effect on turbulence. While several models have been developed to account for the effect of pressure fluctuations (compressibility correction models), very little

\footnotetext{
* Aerospace Engineer, AIAA Associate Fellow

${ }^{\dagger}$ Senior Scientist, AIAA Member
} 
has been done to account for large temperature fluctuations. This has led to poor CFD prediction of non-isothermal flows. For high-temperature jet flow, the standard turbulence models lack the ability to predict the observed increase in growth rate of mixing layer [10-11]. Several researchers [12-17] have modified one or more terms of the transport equations to obtain better agreement in high temperature flows. These modifications affect directly or indirectly the closure terms of the turbulent heat flux $\left(\rho \overline{u_{i} \theta}\right)$ and stresses $\left(\rho \overline{u_{i} u_{j}}\right)$. Theis and Tam [12] changed several coefficients in the turbulent transport equations. However, such extensive modifications of model coefficients completely change the characteristics of the equations and may cause deficiencies in flow prediction accuracy for other problems. Other attempts to sensitize the turbulence model to temperature fluctuations involve more sophisticated closure for the turbulent heat flux term appearing in the average energy equation [15-17]. Explicit algebraic nonlinear heat flux models have also been tested for this purpose. These models have been successful in some fully-developed high temperature turbulent flows. A simpler approach was to model the value of $\mathrm{C}_{\mu}$ as $\mathrm{a}$ function of the total temperature gradient in the flow [18]. The concept behind this approach was to postulate that the large-scale density nonuniformity in the flow would introduce local mixing instability and added turbulence stresses. The modification in [18] was successful in predicting the hot jet mixing rate over a wide range of temperature ratios between the jet and the ambient air. Authors of this paper thought that this variable $\mathrm{C}_{\mu}$ would not be necessary in the multi-scale turbulence model approach because the computations in the code would automatically take care of the large scale density gradient effect.

\section{Approaches}

The governing equations of the time-averaged formulation include the conservation equations for mass, momentum, and energy, and the equation of state. In the present study, the perfect gas law is chosen to represent the air properties, and the eddy-viscosity concept is used to model the Reynolds stresses. The mass, momentum, and energy conservation equations of the time-averaged equations can be written in a conservative form as follows:

$$
\begin{aligned}
& \frac{\partial \rho}{\partial t}+\frac{\partial \rho u_{i}}{\partial x_{i}}=0 \\
& \frac{\partial \rho u_{i}}{\partial t}+\frac{\partial\left(\rho u_{i} u_{j}+p \delta_{i j}\right)}{\partial x_{j}}=\frac{\partial\left(\tau_{i j}-\rho \overline{u_{i} u_{j}}\right)}{\partial x_{j}} \\
& \frac{\partial \rho e_{0}}{\partial t}+\frac{\partial\left(\rho e_{0} u_{i}+p u_{i}\right)}{\partial x_{i}}=\frac{\partial\left(\tau_{i j} u_{j}-\rho \overline{u_{i} u_{j}} u_{j}\right)}{\partial x_{i}}-\frac{\partial\left(q_{i}+C_{P} \rho \overline{u_{i} \theta}\right)}{\partial x_{i}}+\frac{\partial}{\partial x_{i}}\left[\left(\mu_{l}+\frac{\mu_{t}}{\bar{\sigma}_{k}}\right) \frac{\partial k}{\partial x_{i}}\right]
\end{aligned}
$$

In the case of Reynolds Averaged Navier-Stokes (RANS) equations, a Standard Turbulence Model (STM) such as the two-equation $(\mathrm{ke})$ turbulence model is used:

$$
\begin{aligned}
& \frac{\partial \rho k}{\partial t}+\frac{\partial \rho u_{j} k}{\partial x_{j}}=-\rho \overline{u_{j} u_{i}} \frac{\partial u_{i}}{\partial x_{j}}+\frac{\partial}{\partial x_{j}}\left[\left(\mu_{l}+\frac{c_{\mu} k^{2}}{\bar{\sigma}_{k} \varepsilon}\right) \frac{\partial k}{\partial x_{j}}\right]-\rho \varepsilon\left(1 .+M_{\tau}^{2}\right) \\
& \frac{\partial \rho \varepsilon}{\partial t}+\frac{\partial \rho u_{j} \varepsilon}{\partial x_{j}}=-C_{\varepsilon 1} \rho \overline{u_{j} u_{i}} \frac{\partial u_{i}}{\partial x_{j}} \frac{\varepsilon}{k}+\frac{\partial}{\partial x_{j}}\left[\left(\mu_{l}+\frac{c_{\mu} k^{2}}{\bar{\sigma}_{\varepsilon} \varepsilon}\right) \frac{\partial \varepsilon}{\partial x_{j}}\right]-f_{2} \tilde{C}_{\varepsilon 2} \rho \frac{\varepsilon}{k}\left[\varepsilon-v_{l}\left(\frac{\partial \sqrt{k}}{\partial n}\right)^{2}\right] \\
& C_{\mu}=.09, C_{\varepsilon 1}=1.44, \\
& \bar{\sigma}_{k}=\sigma_{k}=1.4, \bar{\sigma}_{\varepsilon}=\sigma_{\varepsilon}=1 \text { and } \overline{C_{\varepsilon 2}}=C_{\varepsilon 2}=1.92
\end{aligned}
$$


$f_{\mu}=\exp \left[\frac{-3.41}{\left(1+\frac{R_{T}}{50}\right)^{2}}\right], R_{T}=\frac{k^{2}}{\mu_{t} \varepsilon}, f_{2}=1 .-0.3 \exp \left(-R_{T}^{2}\right)$

The boundary conditions for $\varepsilon$ and $\mathrm{k}$ at the wall are:

$\varepsilon_{\text {wall }}=v_{l}\left(\frac{\partial \sqrt{k}}{\partial n}\right)^{2}$

$k_{\text {wall }}=0$.

The turbulent stress components are formulated as:

$$
\begin{gathered}
\rho \overline{u_{j} u_{i}}=2 \rho v_{t} S_{j i}-\frac{2}{3} \delta_{j i} \rho k \\
S_{j i}=\frac{1}{2}\left[\frac{\partial u_{j}}{\partial x_{i}}+\frac{\partial u_{i}}{\partial x_{j}}\right]-\frac{1}{3} \delta_{j i} \frac{\partial u_{j}}{\partial x_{i}}
\end{gathered}
$$

For the purpose of this paper, we will define RANS turbulent viscosity as

$$
v_{t}^{R A N S}=f_{\mu} \rho C_{\mu} \frac{k^{2}}{\varepsilon}
$$

\section{A. Two-Stage PANS Approach}

The PANS model [4] was developed to overcome the grid dependency associated with the use of other Hybrid Turbulence Models (HTM). In its original form, PANS [5] replaces the two-equation turbulence model by solving for the unresolved kinetic energy $\mathrm{k}_{\mathrm{u}}$ and the dissipation $\varepsilon_{\mathrm{u}}$. The $\mathrm{k}_{\mathrm{u}}$ equation is identical to the original $\mathrm{k}$ equation. In the $\varepsilon$ equation (equation 2), the following coefficients are used to change the two-equation model to the Hybrid Turbulence Model (HTM), which becomes known as the PANS formulation through the following changes:

$$
\begin{aligned}
& \tilde{C}_{\varepsilon 2}=\frac{f_{k}}{f_{\varepsilon}}\left(C_{\varepsilon 2}-C_{\varepsilon 1}\right)+C_{\varepsilon 1} \\
& \bar{\sigma}_{k}=\frac{f_{k}^{2}}{f_{\varepsilon}} \sigma_{k} \text { and } \bar{\sigma}_{\varepsilon}=\frac{f_{k}^{2}}{f_{\varepsilon}} \sigma_{\varepsilon}
\end{aligned}
$$

where

$$
f_{k}=\frac{k_{u}}{k} \text { and } \quad f_{\varepsilon}=\frac{\varepsilon_{u}}{\varepsilon}
$$


represent the ratios of the unresolved kinetic energy and dissipation to the total kinetic energy and dissipation, respectively. It is only natural to use $\left(f_{k}, f_{\varepsilon}\right)$ to quantify the PANS filter with respect to RANS. Therefore, $\left(f_{k}, f_{\varepsilon}\right)$ are used as the resolution control parameters for PANS. Physics of turbulence dictates that:

$$
\text { ( DNS ) } 0 \leq f_{k} \leq f_{\varepsilon} \leq 1 \quad(\boldsymbol{R A N S})
$$

The original formulation [5] uses constant values for the unresolved kinetic energy parameter $\left(f_{\mathrm{k}}\right)$ and unresolved dissipation rate parameter $\left(f_{\varepsilon}\right)$. The users will select values for these parameters and refine the grid until the flow solution converges toward a solution target. This could be very time consuming for resolving complex threedimensional flows. In the present paper, we will discuss an approach to define the unresolved kinetic energy parameter. Abdol-Hamid and Girimaji [9] introduced a two-stage approach to estimate the values of the unresolved kinetic energy parameter. Here, we will highlight the basic concepts of this approach. Based on a simple dimension analysis, we assume that the turbulent viscosity may be related to the total kinetic energy (k),, $\mathrm{S}$ and $\Delta$ as:

$$
v_{t} \approx \frac{k^{2}}{\varepsilon} \approx \Delta^{2} S \approx \Delta^{2} \frac{\varepsilon}{k}
$$

which leads to

$$
f_{k}^{3} k^{3} \approx \Delta^{2} \frac{k^{3}}{L}
$$

Hence,

$$
f_{k}=C_{h}\left[\frac{1}{\lambda}\right]^{2 / 3}, \lambda=\frac{L}{\Delta}
$$

$\mathrm{C}_{\mathrm{h}}$ is a model coefficient, which needs to be calibrated. In the present paper, a value of one will be used to evaluate the model. Here, we summarize the guidelines to be followed for the sequential two-stage procedure. These guidelines are completely dependent upon flow complexity. For the first stage:

- Complete a three-dimensional or two-dimensional simulation

- Use unsteady or steady calculation; high-order schemes are not required

- Choose a desired level of an allowable RANS turbulence model (one-equation, two-equation, Algebraic Stress, full Reynolds Stress, etc.)

For the second stage:

- Conduct a three-dimensional simulation

- Use unsteady calculation; high-order schemes should be considered

- Use hybrid models (DES, Hybrid RANS/LES, PANS, etc.)

The users need to use the same flow conditions, boundary conditions, and grid resolution for both stages of the procedure.

\section{B. Hybrid RANS/LES Approach}

Nichols and Nelson [2] give an example of a hybrid RANS/LES turbulence model. This method was implemented in conjunction with Menter's SST two-equation turbulence model and is termed a multi-scale (MS) model. In the present paper, this hybrid model is used with the two-equation model described in equations 2 and 3. The turbulent length scale, used in this implementation, is defined as: 


$$
l_{t}=\max \left(6 \cdot \sqrt{v_{t} / \Omega}, k^{3 / 2} / \varepsilon\right)
$$

The sub grid turbulent kinetic energy is defined as

$$
k^{L E S}=f_{d} k
$$

The damping function is defined as

$$
f_{d}=\{1 .+\tanh [2 \pi(\Lambda-0.5)]\} / 2
$$

where,

$$
\Lambda=\frac{1}{1+\left[\frac{l_{t}}{\Delta}\right]^{4 / 3}}=\frac{1}{1+\lambda^{4 / 3}}
$$

$\lambda$ is the unresolved characteristic ratio, and

$$
\Delta=\max \left(\Delta_{x}, \Delta_{y}, \Delta_{z}\right)
$$

The eddy viscosity is then calculated from:

$$
\begin{aligned}
& v_{t}=f_{d} v_{t}^{R A N S}+\left(1 .-f_{d}\right) v_{t}^{L E S} \\
& v_{t}^{L E S}=\min \left(v_{t}^{R A N S}, 0.084 \Delta \sqrt{k^{L E S}}\right)
\end{aligned}
$$

Note that this hybrid model allows the transition from RANS to LES as a function of the local grid spacing and the local turbulent length scale predicted by the RANS model rather than as a function of the grid spacing alone. This allows the model to detect whether it can resolve the turbulent scales present on the existing grid before its transition over to the LES mode.

\section{Results and Discussions}

Two test cases were selected to evaluate the present modifications. The first test case is a single subsonic jet operated at design pressure condition. We selected this case to compare RANS, PANS and LES approaches in simulating subsonic jet. This case provides the calibration of the PANS approach. The second test case is a multistream subsonic jet configuration. This case addresses the complexity of modeling multi-stream flows. We used a 0.03 time step based on the radius of the nozzle and the free stream acoustic speed. We ran 4000 iterations to initiate the unsteadiness of the jet flow. Then, the solutions were averaged over the last 20,000 iterations. It was observed that approximately 4 sub-iterations per physical time step produced the optimal convergence per iteration. However, the physics of the specific problem will dictate the sub-iteration number for other cases. In the present results, four sub-iterations typically reduced the residual by two orders of magnitude at that time level, with no improvement using more iteration.

\section{A. High-Temperature Single Nozzle Jet Flow}

The present study used the benchmark experiments performed by Bridges [19] at the NASA Glenn Laboratory with the flow condition as indicated in Table 1, for core, and free stream. 
Table 1: Experimental Subsonic Condition

\begin{tabular}{|l|l|l|l|}
\hline & $\begin{array}{l}T_{t} \\
{\left[{ }^{\circ} R\right]}\end{array}$ & $\begin{array}{l}P_{t} \\
{[p s i]}\end{array}$ & $M$ \\
\hline Core & 1400 & 17.68 & 0.55 \\
\hline Free Stream & 540 & 14.3 & 0.01 \\
\hline
\end{tabular}

The computational mesh is a full three-dimensional grid with 120 cells in the circumferential direction. The computational domain is divided into 48 blocks. The super fine mesh has a total of 4,000,000 cells. Grid points are clustered near the solid surfaces and around the shear layer. We used a uniform streamwise grid spacing for $2<$ $x / D j<12$. The value of $y^{+}$for the first cell off the surface varied between 0.2 and 2. During the course of this simulating this case, we tried single and double precision calculations. We found no significant difference in the results. Also, we tried 4, 8 and 12 sub-iterations for the dual step time accurate approach. We also found no significant difference in the results. Based on these results, we will use single precision and 4 sub-iterations in all of the presented results in this paper.

First, we used the RANS formulation to get time-averaged quantities to calculate the characteristic length-scale ratio. This ratio varies in space and is used to produce the unresolved kinetic energy parameter $\left(f_{k}\right)$. Figure 1a shows the distribution of this function. This parameter identifies the RANS and PANS regions. The RANS regions are defined with the parameter set at a value of one. The PANS regions are the remaining flow domains where $f_{k}$ values are less than one. We use this parameter in solving the PANS formulation. We calibrate the medium grid $(1,000,000$ cells) to get the velocity profile to closely resemble the experimental data for the velocity distribution. We found that $\mathrm{C}_{\mathrm{h}}$ should be 1.05 for the present test case. All the calculations performed hereafter used the same value of $\mathrm{C}_{\mathrm{h}}=1.05$.

Figure $1 \mathrm{~b}$ shows the snapshot of a two-dimensional on the X-Y plane for the velocity $(u / U j)$ using the PANS formulation. The result shows the unsteady behavior of the jet flow as it interacts with the external flow. A similar observation was found as the RANS/LES formulation was used. In the case of RANS prediction, there was not a significant difference between the snapshot and the time-averaged flow quantities. In this case, the snapshot solution is similar to the one shown in Figure 2. This was caused by the fact that RANS over predicts the eddy viscosity, resulting in excessive damping of unsteady motion. Consequently, the eddy viscosity attains un-physically large values due to unresolved scales, and suppresses most temporal and spatial fluctuations in the resolved flow field.

Figure 2 shows the two-dimensional slice of the time-averaged results of using RANS and PANS formulations, as compared with experimental data. There is no significant difference between the time-averaged and snapshot of the unsteady RANS result. The PANS results and similarly RANS/LES (not shown) produce good comparison with experimental data. Figure 3 shows the comparison of the jet centerline velocity using RANS, RANS/LES and PANS formulations as compared with experimental data. Figure 4 shows the jet centerline turbulent kinetic energy. Both PANS and RANS/LES overpredict the total turbulent kinetic energy. We define total kinetic energy, $k_{t}$ as:

$$
k_{t}=k_{r}+k_{u}=\frac{1}{2}\left[\overline{u_{i} u_{i}}-\overline{u_{i}} \overline{u_{i}}\right]+k_{u}
$$

In the case of the RANS results, the resolved kinetic energy, $k_{r}$ is zero. Both RANS/LES and PANS provided a mechanism for the RANS equations to resolve the largest scales of motion. Figure 5 shows the reduction of the unresolved kinetic energy, $k_{u}$, as compared with total value. In general, the RANS produces the same values for all three normal stress components. Figure 6 shows the comparisons and distributions of the normal stress components $(u u, v v$, and $w w)$ using PANS. In this case, PANS produced different values for the three normal stress components with a trend similar to the experimental data. The levels of the vv and ww components are very close to the experimental data. The predicted uu component is much higher than the experimental data. DeBonis [20] reported the same observation that the LES simulation produced a much higher value of uu as compared with $\mathrm{M}=0.9$ hot jet experimental data.

We attempted to provide grid convergence study for the single jet case. We tried three grid levels; $1,000,000$ cells (medium), 2,000,000 cells (fine) and 4,000,000 cells (super fine). In this case, the grid is refined in the flow direction while holding the filter coefficient $C_{h}$ (see equation 8) constant. This causes the filter width to be refined along with the grid. The smallest value of $f_{\mathrm{k}}$ was around 0.1 . Under such a procedure the solution converges to a DNS, since the filter width approaches zero along with the grid spacing. As a result this approach attempts to calculate different turbulent scales explicitly, whether or not the numerical resolution is sufficient to accurately compute those scales. The result of this study is shown in Figure 6. The medium and fine grid provides reasonable grid convergence. However, the super fine grid completely diverges from the other solutions. This is due to 
limitations of the numerical scheme to handle such flow physics. In such case, a higher order scheme could be more effective.

Now, we freeze the value of $f_{\mathrm{k}}$ from the fine grid solution and use it for the super fine grid. Figure 7 shows the comparisons of this approach with fine and super fine grids. The result of this approach improves the super fine grid solution and now it matches the fine grid solution. This equivalent is to use $f_{\varepsilon}$ of value 0.85 instead of 1.0.

\section{B. High-Temperature Multi-stream Nozzle Jet Flow}

The second test configuration includes a separate fan and core nozzle flows at a bypass ratio of five with an external plug. One set of data was selected from the reported test results [21], with the flow condition as indicated in Table 2 for core, fan and free stream.

Table 2: Experimental Subsonic Condition

\begin{tabular}{|l|c|c|c|}
\hline \multicolumn{4}{|c|}{ (** please compute the Mach numbers for core and fan) } \\
& $\begin{array}{l}T_{t} \\
{\left[{ }^{\circ} \mathrm{R}\right]}\end{array}$ & $\begin{array}{l}P_{t} \\
{[\mathrm{psi}]}\end{array}$ & $M$ \\
\hline Core & 1498 & 21.72 & 0.8 \\
\hline Fan & 647 & 24.36 & 0.9 \\
\hline Free Stream & 530 & 14.7 & 0.28 \\
\hline
\end{tabular}

This test configuration was part of a comprehensive investigation on jet exhaust noise due to the pylon-chevronjet interaction [11], which was tested at NASA. As previously discussed, the present PANS approach was tuned on the axisymmetric grid to the round nozzle experimental results. For the limited space and scope of this paper, we will discuss the comparison with only the axisymmetric configuration shown in Figure 9.

The computational domain for the solution extended from $x / D_{c}=-6.3$ to $x / D_{c}=31.6$ in the axial direction and $6.3 D_{c}$ in the radial direction, where $D_{c}$ is the diameter of the baseline core nozzle, $12.80 \mathrm{~cm}$. The origin, $x / D_{c}=0.0$ was set at the exit of the fan nozzle so that the exit of the core nozzle is at about $x / D_{c}=0.5$. The computational mesh is a three-dimensional shaped grid with 120 cells in the circumferential direction. The computational domain is divided into 92 blocks. The mesh has a total of 7,750,000 cells. Grid points are clustered near the solid surfaces and around the shear layer. The value of $\mathrm{y}^{+}$for the first cell off the surface varied between 0.16 and 1.8.

Computational solutions were obtained for RANS, hybrid RANS/LES and PANS formulations. The simulated conditions were set to coordinate with the data presented in Ref [11]. The computational results were conducted for a free stream Mach number of 0.28 . For the PANS calculations, we used $C_{h}=1.05$ and $C_{h}=0.95$ to study the sensitivity of the prediction to the variation of $\mathrm{C}_{\mathrm{h}}$.

Comparison between computed stagnation temperatures at the symmetry plane shows that both RANS/LES and PANS produced faster mixing than RANS solution as shown in Figure 10. Comparison between computed stagnation temperature and experimental data is shown in Figures 11 and 12. The RANS solution produces slower mixing than experimental data. On the other hand, RANS/LES produces reasonably good agreement with the data. In Figure 11, the calculations were compared with the experimental centerline values. The RANS approach was not able to match the experimental data accurately while both RANS/LES and PANS were in reasonably good agreement with the experimental values. We also found that the PANS solutions are sensitive to the variation of $C_{h}$. As shown in Figure 12, RANS/LES was able to more accurately predict the temperature flow field and produce a result closer to the experimental data while the RANS approach over predicted the temperature in the core region. The result from PANS was in reasonably good agreement with experimental data.

\section{Summary and Future Work}

The hybrid RANS/LES and PANS turbulence models are relatively new and will need to be exercised for a wide variety of problems to determine their accuracy, before they become an accepted tool for fluid dynamics modelers. They seem to offer much for unsteady flow applications, but issues such as grid sensitivity need to be further addressed. Hopefully more effort will go into these models in the near future, so that they can be matured for use in everyday applications. The new capabilities have the potential to improve the accuracy and robustness of creating a simulation of an unsteady flow field. This new class of turbulence models is inherently grid size dependent, since increasing the grid resolution allows smaller and smaller turbulent scales to be resolved. 
We have introduced and implemented a novel two-stage procedure to efficiently estimate the level of scale resolution possible for a given flow on a given grid for Partial Averaged Navier-Stokes (PANS) and other hybrid models. This is a two-stage procedure. In the first stage, a RANS simulation with a Standard Turbulence Model (STM) such as $k \varepsilon$ is used to produce an estimate of $f_{\mathrm{k}}$ over the entire grid domain. In the second stage, we supply $f_{\mathrm{k}}$ for the selective application of a Hybrid Turbulence Model (HTM) such as the PANS formulation in regions where the grid density is sufficient to resolve a portion or all of the large-scale flow structures. In the present implementation, $f_{\mathrm{k}}$ is a function of length-scale and grid size that represents a characteristic length-scale ratio.

In the present paper, we selected the subsonic high-temperature jet flows to calibrate and validate the PANS approach. This implementation is a first step towards adding a variable resolution turbulence model capability to CFD codes. The PAB3D code can now be used to refine the PANS formulation and to conduct validation computations using a variety of simple and complex flow physics problems. This approach needs to be calibrated, verified and validated for a wide range of flow problems such as different temperature jet, cavity and others.

\section{Acknowledgments}

This work is part of a larger ongoing study of complex nozzle configurations. A jet noise prediction method based on computational information is also being pursued together with the computational results and the experiments. The authors would like to thank Dr. Russ Thomas, Dr. S. Paul Pao, Dr. Craig Hunter from NASA Langley Research Center, and Dr. Steve Massey from Eagle Aeronautics for long hours of fruitful discussions. The second author would like to acknowledge the support of NASA Langley Research Center for providing the funding needed to carry out this work.

\section{References}

1. Spalart, P. R., "Young Person's Guide to Detached Eddy Simulation Grids," NASA CR-2001, 211032, 2001.

2. Nichols, R.H. \& Nelson, C.C., “Application of Hybrid RANS/LES Turbulence Models” AIAA 2003-0083, 2003.

3. Mani, M., Paynter, C.C., "Hybrid Turbulence Models for Unsteady Simulation of Jet Flows." AIAA 20022959, 2002.

4. Batten, P., Goldberg, U., and Chakravarthy, S., "LNS - An Approach Towards Embedded LES," AIAA Paper 2002-0427, 2002.

5. Girimaji S., Sreenivasan, R., Jeong E., "PANS Turbulence Model For Seamless Transition Between RANS, LES: Fixed-Point Analysis and Preliminary Results." FEDSM2003-45336, Proceedings of ASME FEDSM'03 2003 4th ASME-JSME Joint Fluids Engineering Conferences, July 13-16, Honolulu, Hawaii USA, 2003.

6. Abdol-Hamid, K., "Development of Three-Dimensional Code for the Analysis of Jet Mixing Problem," NASA CR 4200, 1988.

7. Massey, S.J., \& Abdol-Hamid, K.S., "Enhancement and Validation of PAB3D for Unsteady Aerodynamics," AIAA Paper 2003-1235, 2003.

8. PAB3D Code Manual Originally Developed by the Propulsion Aerodynamics Branch, now under a cooperative program between the Configuration Aerodynamics Branch, NASA Langley Research Center and Analytical Services \& Materials, Inc. Hampton, VA. See http://www.asm-usa.com/software/PAB3D.

9. Abdol-Hamid, K., and Girimaji, S. "A Two-Stage Procedure Toward the Efficient Implementation of PANS and Other Hybrid Turbulence Models," to be published as NASA TM-213260, 2004.

10. Seiner, J.M., Ponton, M.K., Jansen, B. J., and Lagen, N. T., "The Effects of Temperature on Supersonic Jet Noise Emission," DGLR/AIAA 14th Aeroacoustics Conference, Aachen, Germany, AIAA Paper No. 9202-046, May 1992. 
11. Thomas, R.H., Kinzie, K.W. and Pao, S. Paul, "Computational Analysis of a Pylon-Chevron Core Nozzle Interaction,” AIAA Paper 2001-2185, May 2001.

12. Theis, A. T., and Tam, C. K. W., "Computation of Turbulent Axisymmetric and Non-axisymmetric Jet Flows Using the K-ع Model,” AIAA Journal, Vol. 34, No. 2, February 1996, pp. 309-316.

13. Tam, C. K.W., and Ganesan, A., "A Modified k- $\varepsilon$ Model for Calculating the Mean Flow and Noise of Hot Jets," AIAA 2003-1064, January 2003.

14. Lebedev A. B., Lyubimov A. D., Maslov, V. P., Mineev, B. I., Secundov, A. N., and Birch, Stanley F., "The Prediction of Three-Dimensional Jet Flows for Noise Applications," AIAA 2002-2422, 2002.

15. So, R. M. C., and Sommer, T. P., "An Explicit Algebraic Heat-Flux Model for the Temperature Field," International Journal of Heat and Flow, 1995, Vol. 7, pp. 455-465.

16. Ronki, M., and Gatski, Thomas B., "Predicting Turbulent Convective Heat Transfer in Fully Developed Duct Flows," International Journal of Heat and Flow, Vol. 22, 2001, pp. 381-392.

17. Abe, K., Kondoh, T., and Nagano, Y., “A Two-equation Heat Transfer Model Reflecting Second-Moment Closures for Wall and Free Turbulent Flows," International Journal of Heat and Flow, Vol. 17, 1996, pp. 228-237.

18. Abdol-Hamid, K., Pao, S., Massey, S., and Elmiligui, A. "Temperature Corrected Turbulence Model for High Temperature Jet Flow,” ASME Journal of Fluids Engineering, Vol. 126, No 5, 2004.

19. Bridges, J. and Brown, C., "Parametic Testing on Single Flow Hot Jet," AIAA 2004-2824, 2004.

20. DeBonis, J., “A Large-Eddy Simulation of a High Reynolds Number Mach 0.9 Jet,” AIAA 2004-3025, 2004.

21. Massey, S.J., Thomas, R.H., Abdol-Hamid, K.S and Elmiligui, A., "Computational and Experimental Flowfield Analyses of Separate Flow Chevron Nozzles and Pylon Interaction," AIAA-2003-3212, 2003. 


\section{$f_{k}=1$ RANS Regions}

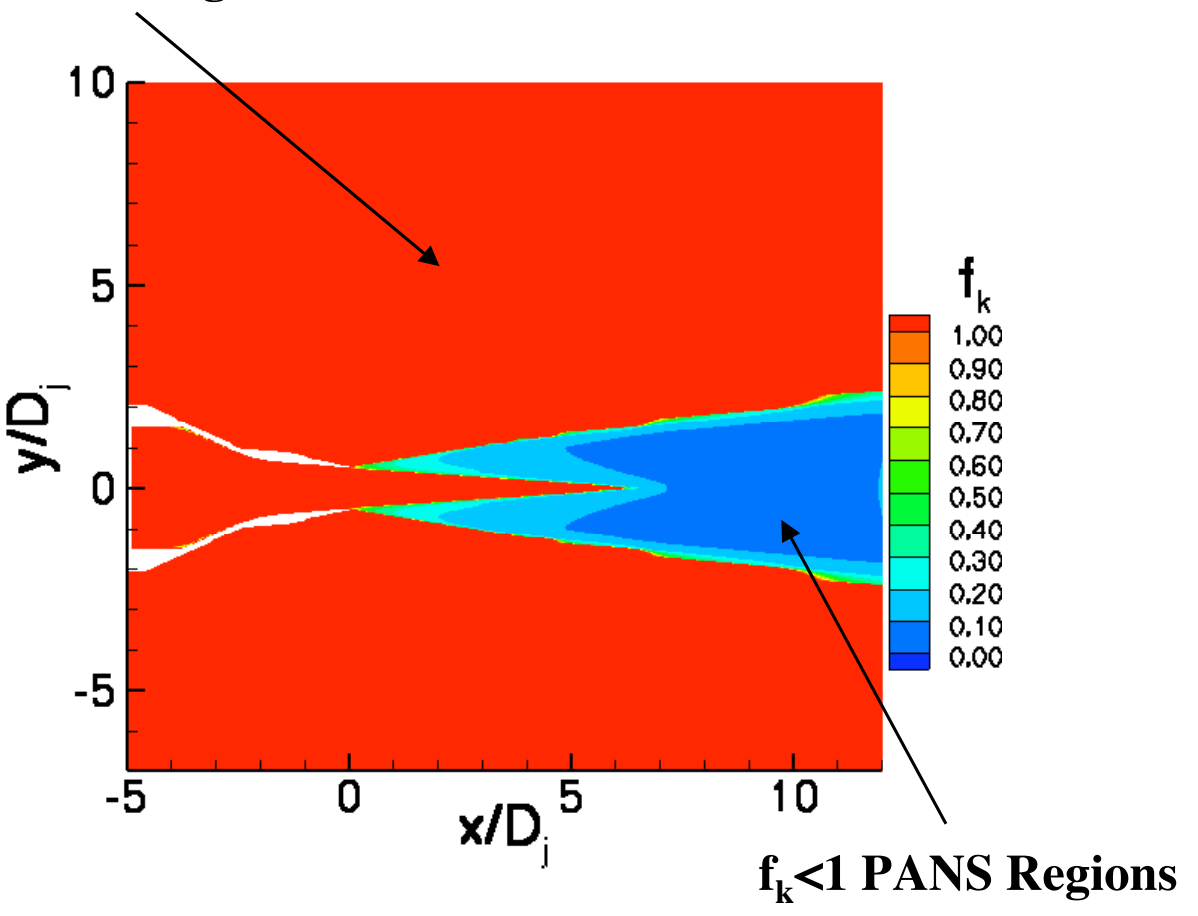

a) Unresolved Turbulent Kinetic Energy Parameter, $f_{k}$ Contours.

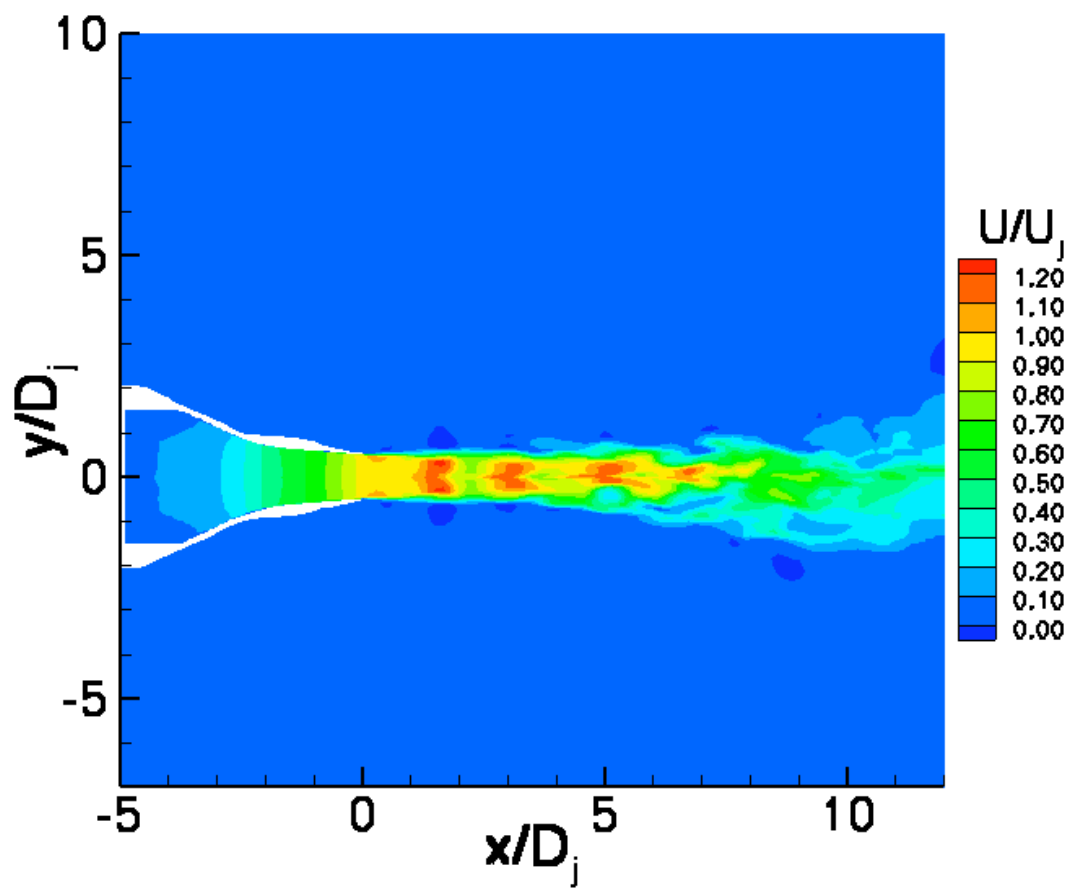

b) Snapshot of Velocity Contour

Figure 1. Single Nozzle Predictions using PANS Formulation 
DATA

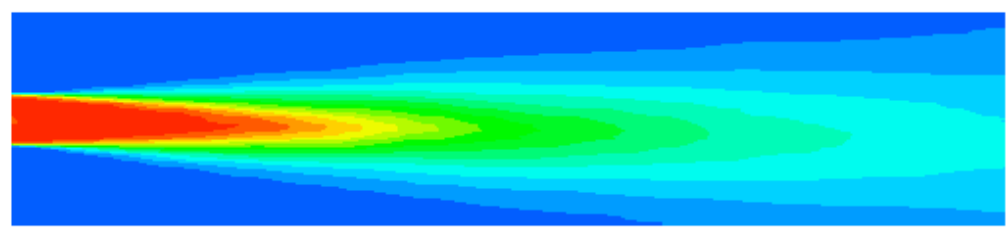

PANS

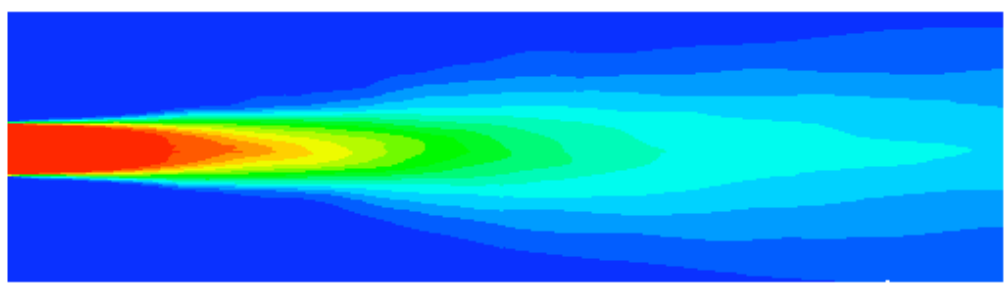

RANS

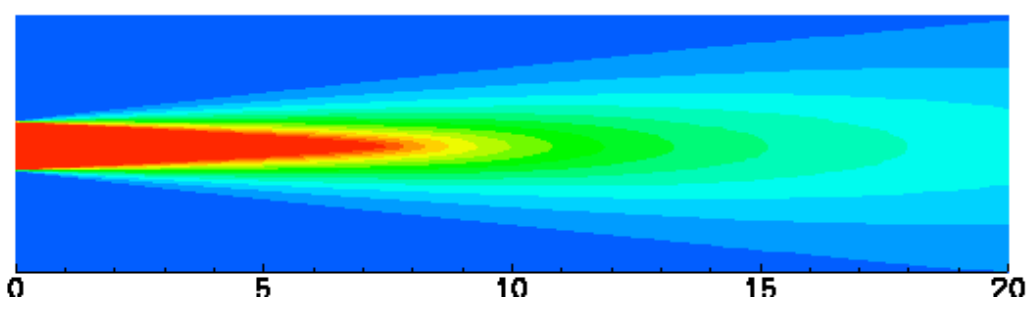

Figure 2. 2D Time Averaged Velocity Contour Results from RANS, and PANS Formulations as Compared with Experimental Data. 


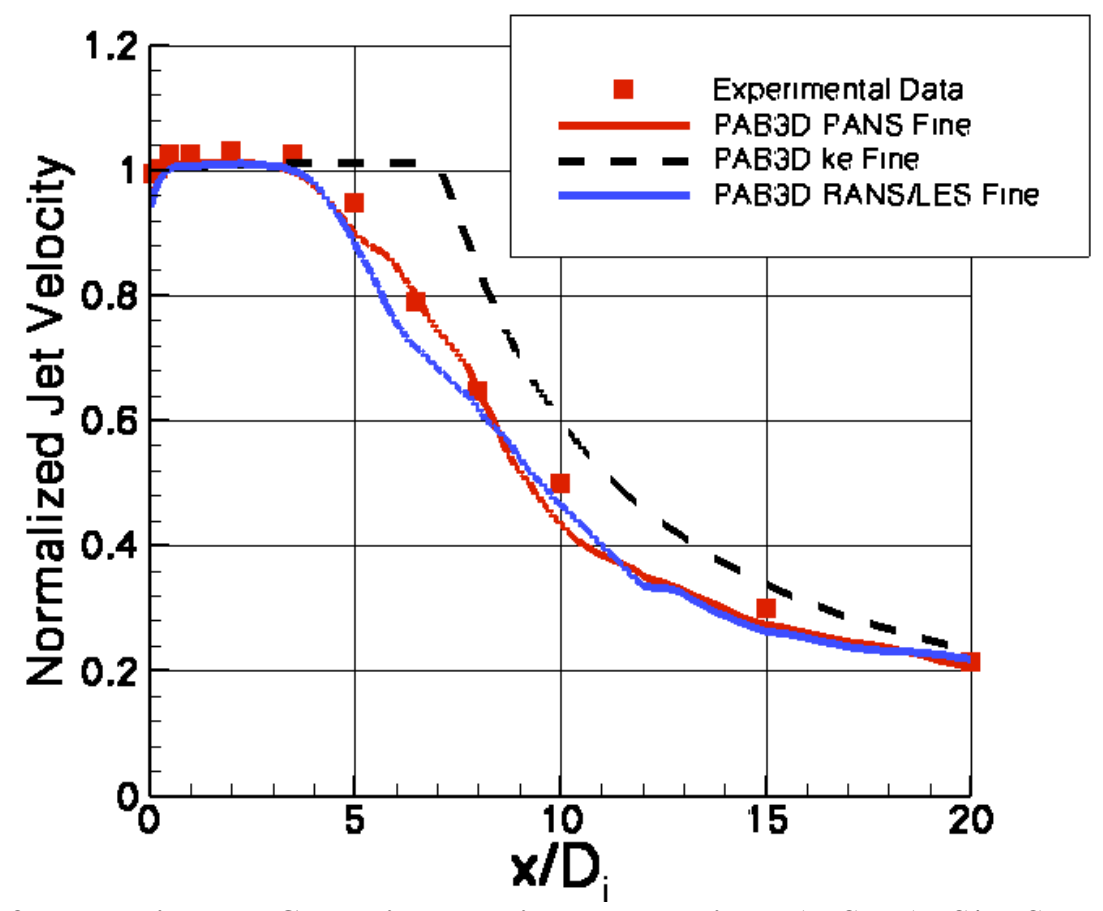

Figure 3. Normalized Jet Centerline Velocity Results using RANS, RANS/LES and PANS Formulations as Compared with Experimental Data.

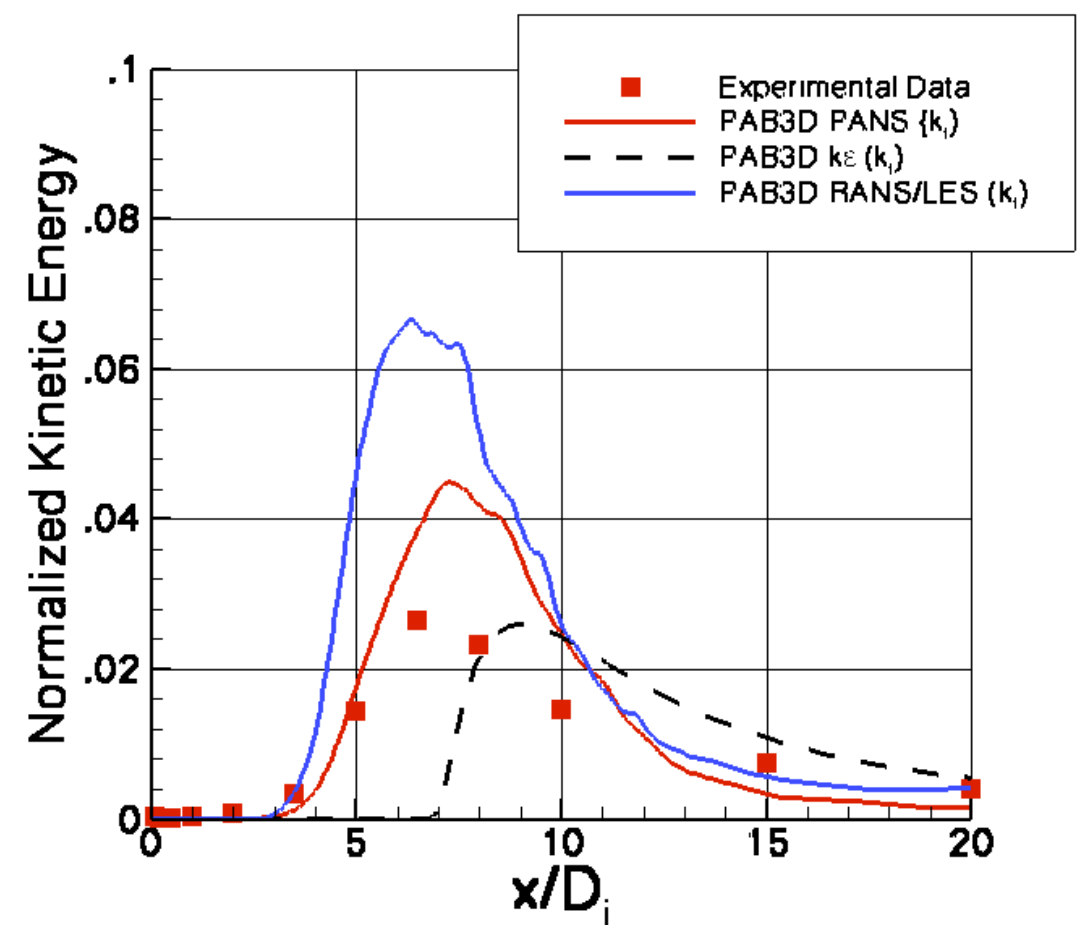

Figure 4. Normalized Jet Centerline Turbulent Kinetic Energy Results using RANS, RANS/LES and PANS Formulations as Compared with Experimental Data. 


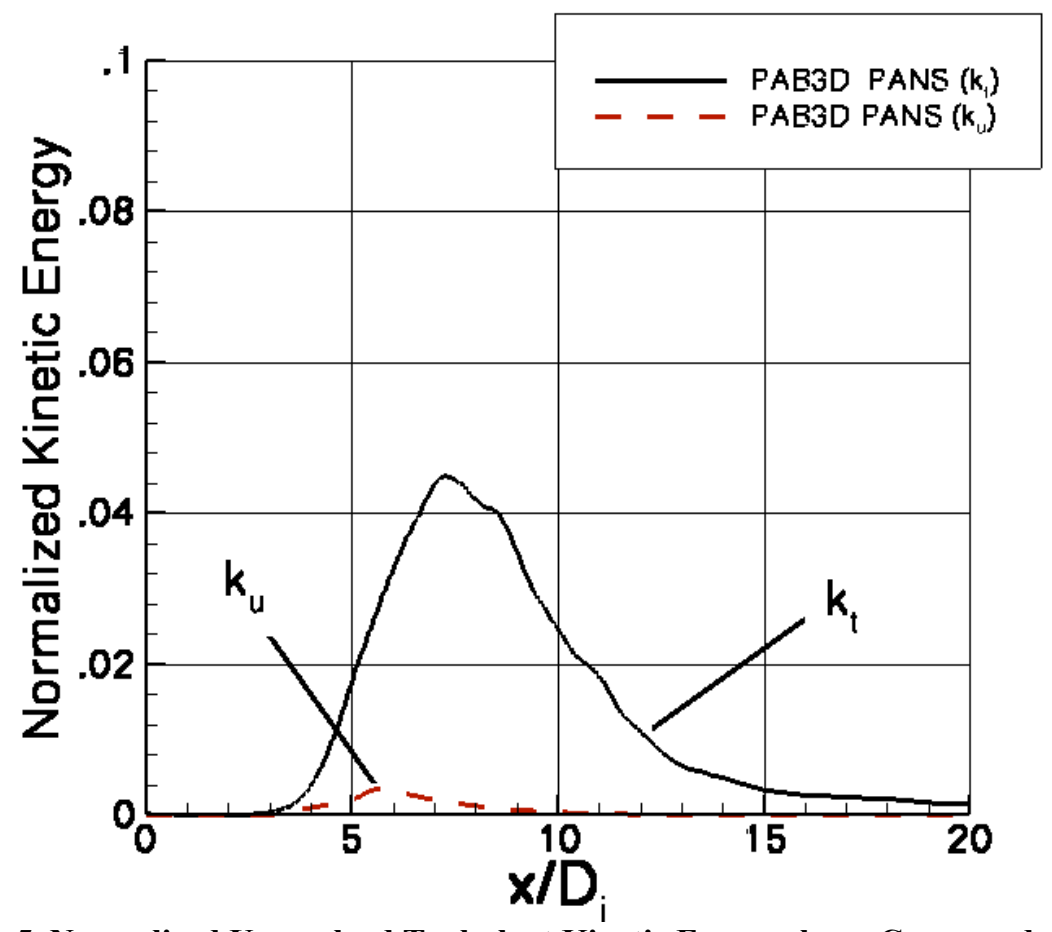

Figure 5. Normalized Unresolved Turbulent Kinetic Energy, $k_{u}$ as Compared with Total Kinetic Energy, $k_{t}$ using PANS.

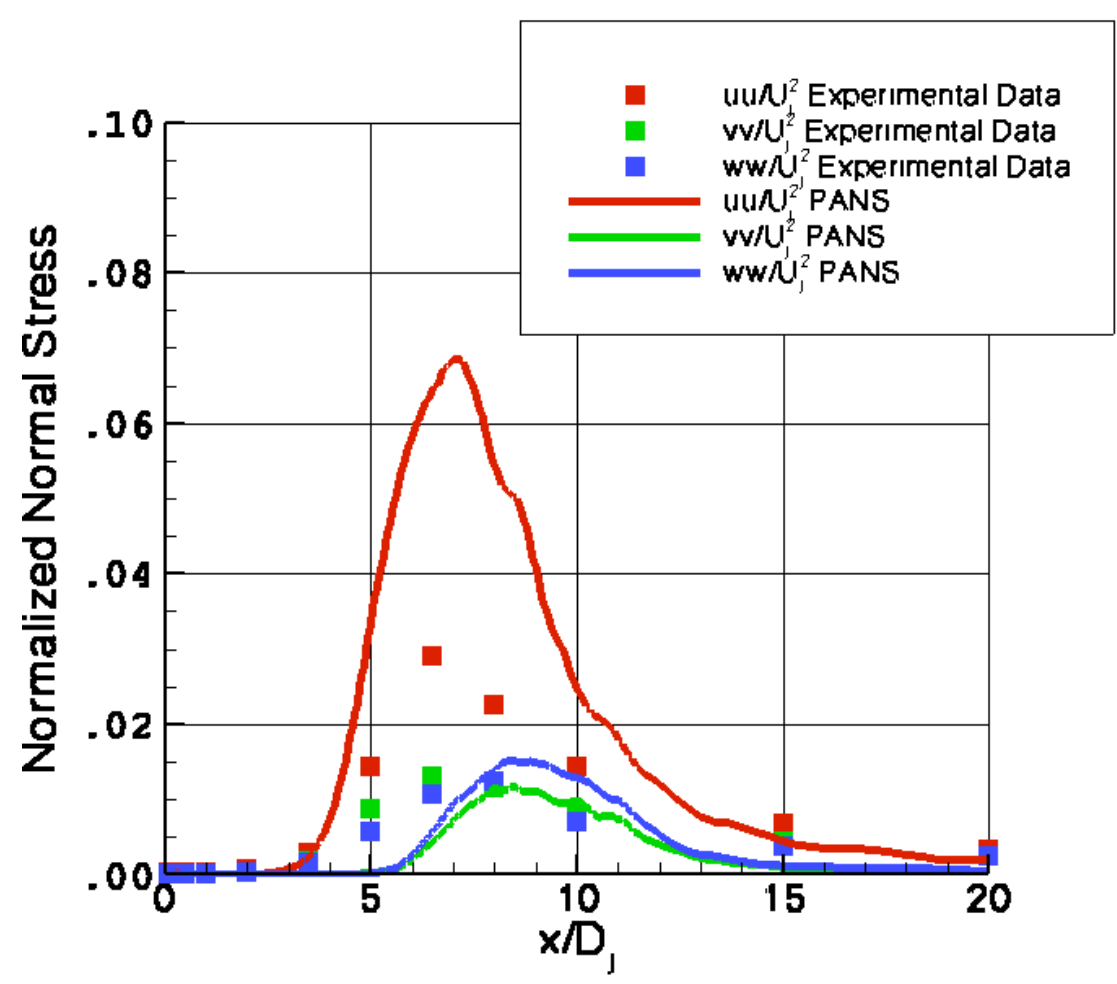

Figure 6. Normalized Normal Stress Components (uu, vv, ww) as Compared Experimental Data. 


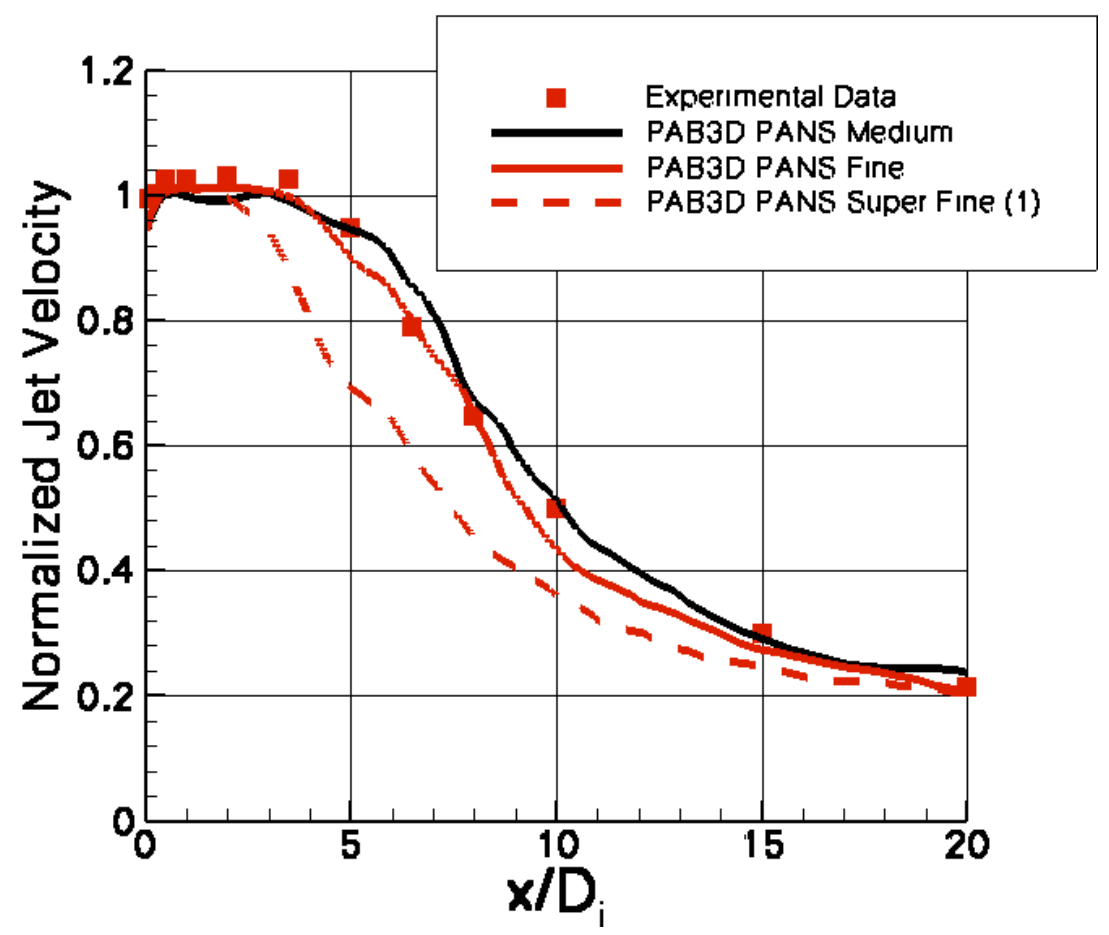

Figure 7. Normalized Jet Centerline Velocity Results using PANS Formulation as Compared with Experimental Data.

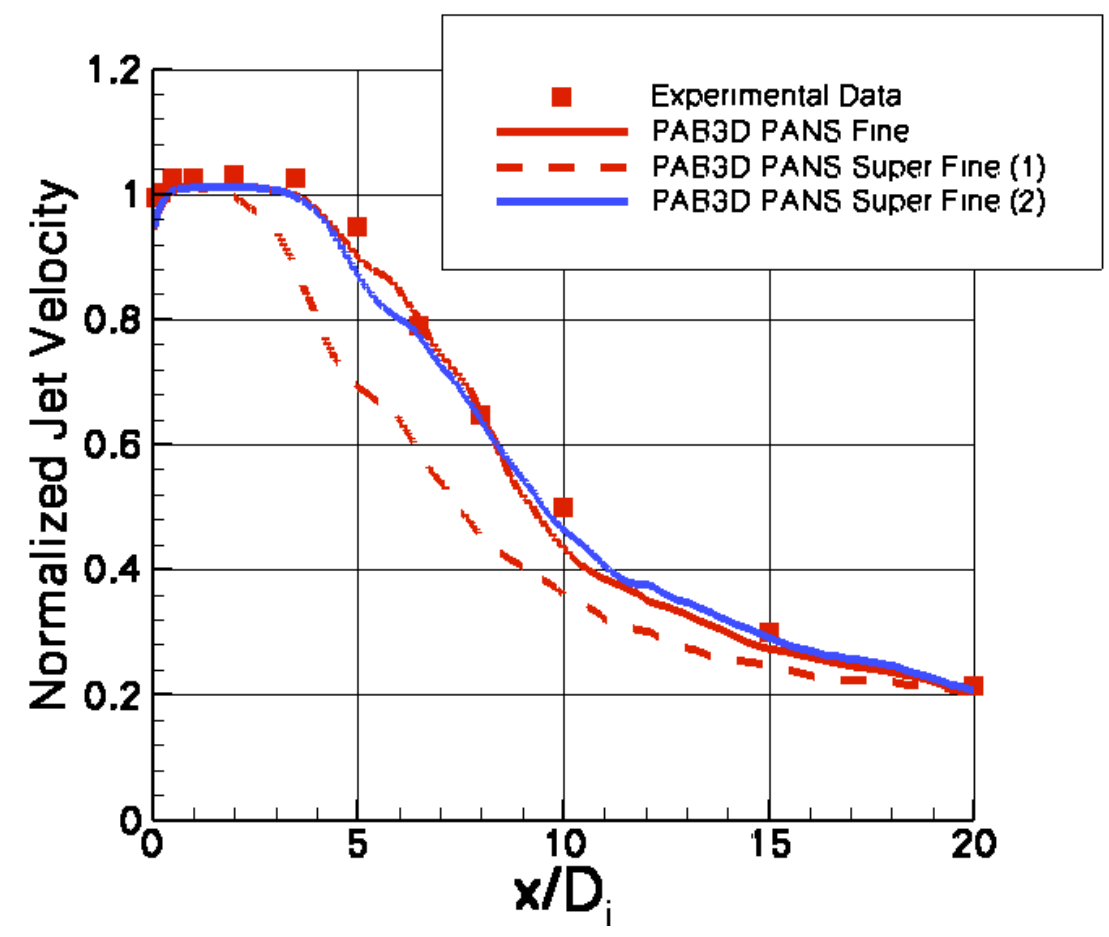

Figure 8. Normalized Jet Centerline Velocity Results using PANS Formulation as Compared with Experimental Data. 


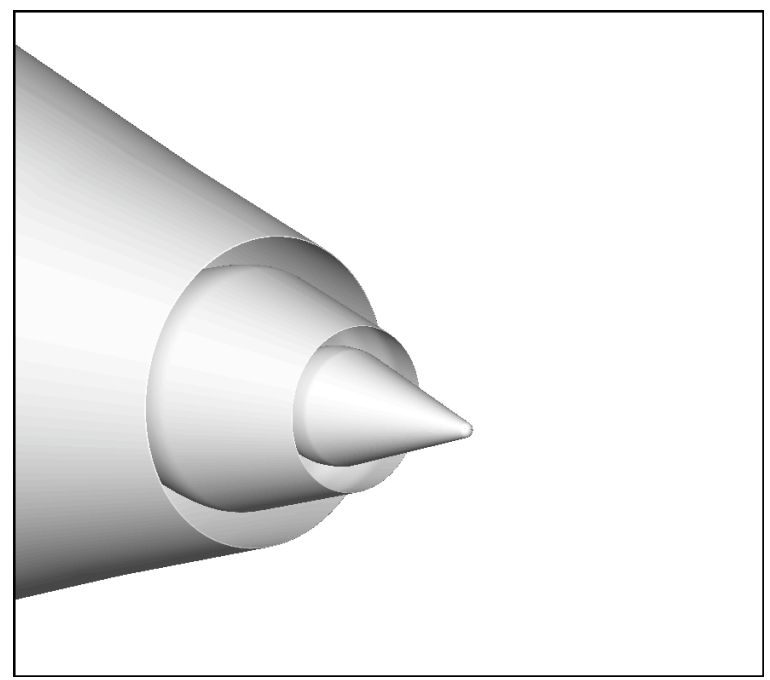

Figure 9. Configuration 1, Baseline Round Core Nozzle with Fan Nozzle.

RANS/LES

PANS

RANS

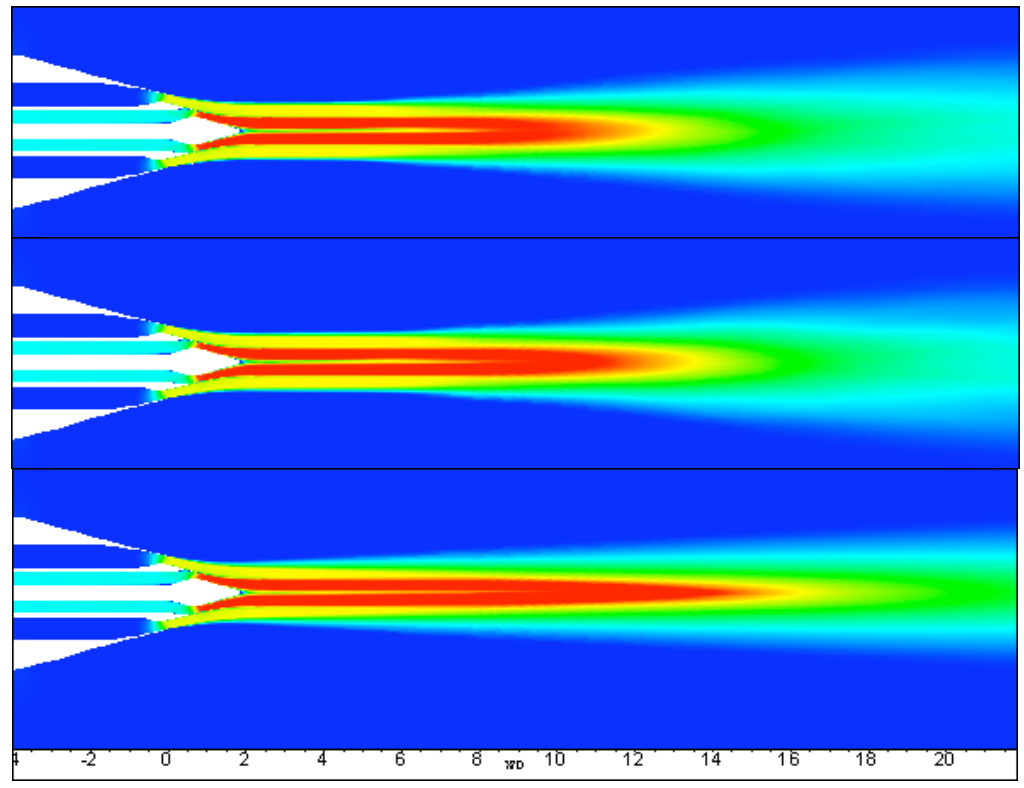

Figure 10 Velocity contours on symmetry plane 


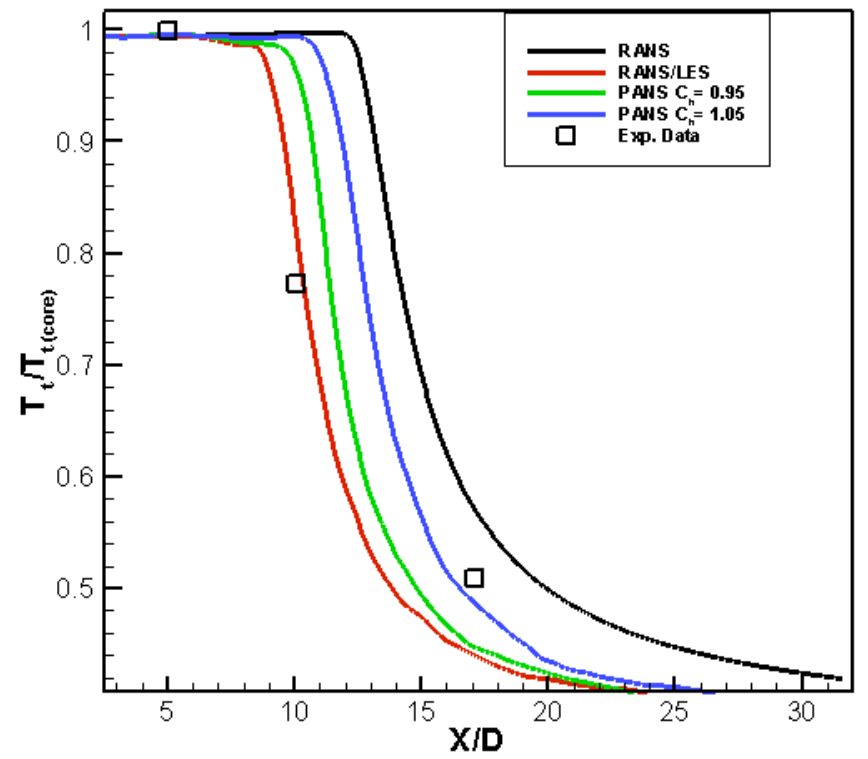

Figure 11. Comparisons of Computed Normalized Centerline Stagnation Temperature and Data [11]. 

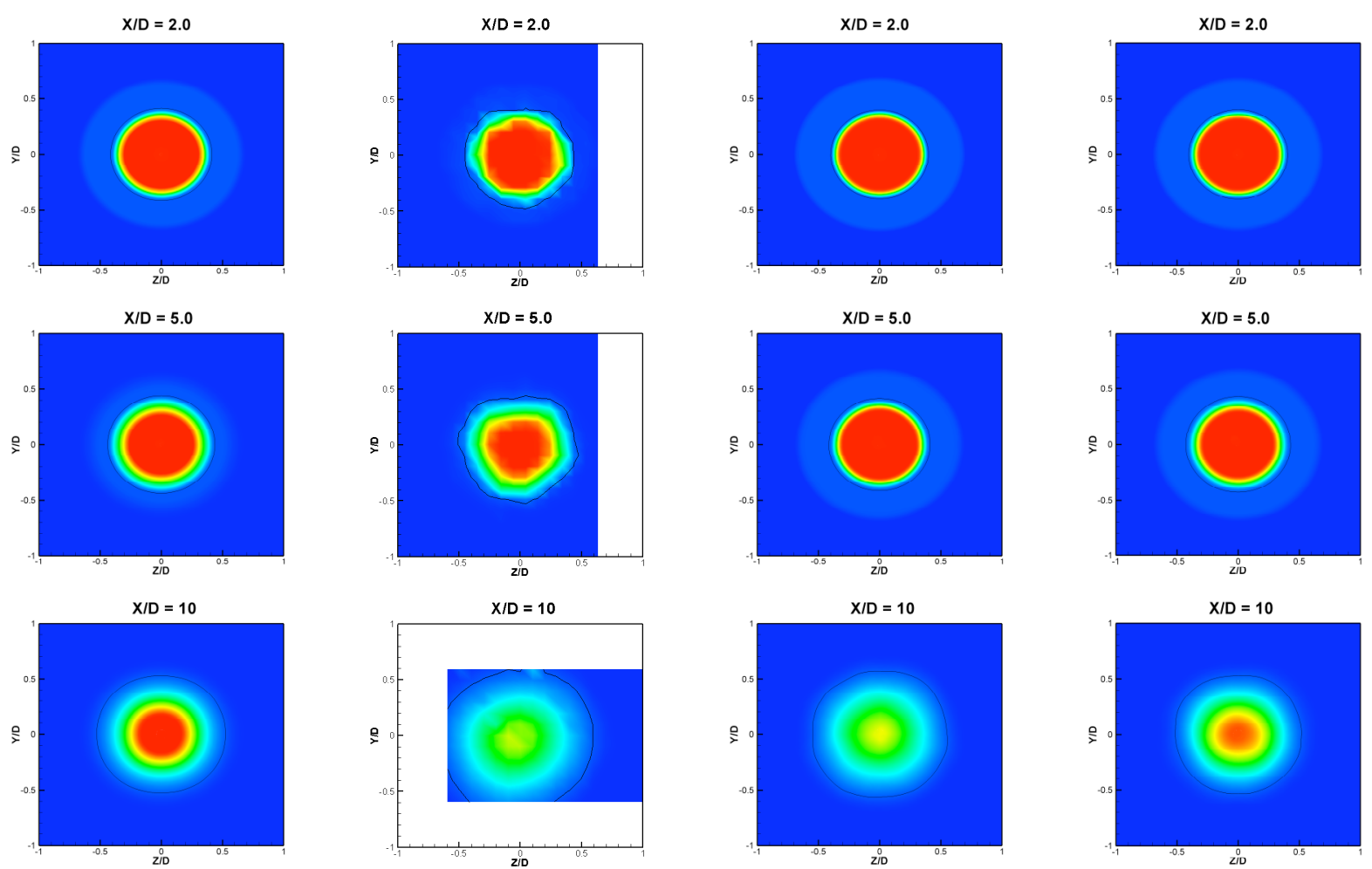

$X / D=17.0$

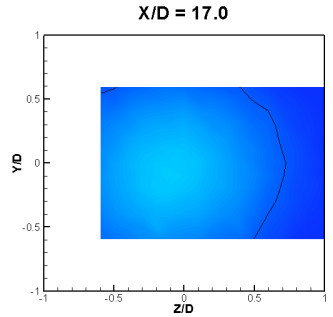

$\mathrm{X} / \mathrm{D}=17.0$

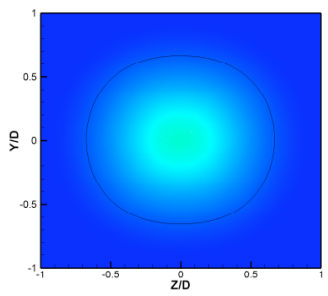

$\mathrm{k} \varepsilon$

Data [11]

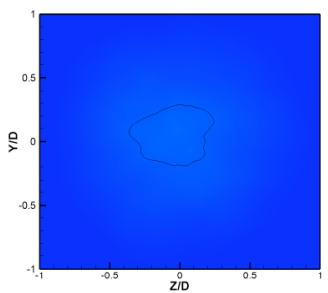

RANS/LES

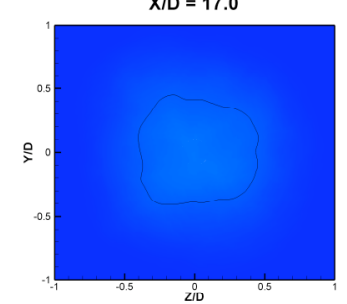

PANS $\mathrm{C}_{\mathrm{h}}=0.95$

Figure 12. Comparison of the stagnation temperature prediction with data [11] 